\title{
On some new variations of Hardy type inequalities
}

Zareen A. Khan

Department of Mathematics, Princess Nora Bint Abdul Rahman University, Riyadh, KSA.

Communicated by $\mathrm{X}$. J. Yang

\begin{abstract}
The goal of this paper is to establish some new variations of the inequalities which originate from the well-known Hardy type inequalities. The method applied in this paper to achieve our results is related to the idea used by Levinson to obtain the generalizations of Hardy's integral inequality. (c)2017 All rights reserved.
\end{abstract}

Keywords: Hardy type inequality, Hölder's inequality.

2010 MSC: 426D15.

\section{Introduction}

In [1], Hardy established the following inequality: if $p>1, f(x)>0$ for $0<x<\infty$, and

$$
R(x)=\frac{1}{x} \int_{0}^{x} F^{p}(x) d x
$$

then

$$
\int_{0}^{\infty} R^{p}(x) d x<\left(\frac{p}{p-1}\right)^{p} \int_{0}^{\infty} F^{p}(x) d x
$$

unless $f=0$. The constant is the best possible.

The following variation of (1.1) has been proved by Izumi and Izumi [3]: if $p>1$, and $s<-1$ and let $f$ be a nonnegative and integrable function on $(0, \pi)$, if $x^{s} f^{p}(s)$ is integrable, then

$$
\int_{0}^{\pi} x^{s} G^{p}(x) d x \leqslant\left(\frac{p}{-s-1}\right)^{p} \int_{0}^{\pi}\left|f \frac{x}{2}-f(x)\right|^{p} d x
$$

where

$$
G(x)=\int_{\frac{x}{2}}^{x} \frac{1}{t} f(t) d t .
$$

The alternative proofs and extensions have been studied by many authors due to the importance of Hardy type inequalities, see [1-9]. Our results are the variations of Hardy inequality (1.1) given by Levinson [4] and the inequality (1.2) given by Izumi and Izumi [3].

Email address: faascsm@ku.ac.th (Zareen A. Khan)

doi:10.22436/jnsa.010.04.33 


\section{Main results}

Throughout in this paper, we assume that all the integrals exist in their respective domains of their definitions.

Theorem 2.1. Let $\mathrm{p}_{\mathrm{r}}>1: 1 \leqslant \mathrm{r} \leqslant \mathrm{n}, \mathrm{m}>1, \mathrm{q}>0$ be a constant and $\mathrm{f}_{\mathrm{r}}$ be a nonnegative and integrable function on $(0, \mathrm{~b}): 0<\mathrm{b}<\infty$. Also, let $w_{\mathrm{r}}$ be a positive and absolutely continuous function on $(0, \mathrm{~b})$ and

$$
1+\frac{2 q_{r} x}{m-1} \frac{w^{\prime}(x)}{w(x)} \geqslant \frac{1}{\alpha_{r}} ; 1 \leqslant r \leqslant n
$$

for almost all $\mathrm{r} \in(0, \mathrm{~b})$ and for some $\alpha_{\mathrm{r}}>0$. If $\mathrm{F}_{\mathrm{r}}$ is defined by

$$
F_{r}(x)=\int_{\frac{x}{2}}^{x} \frac{1}{t}\left(\int_{\frac{t}{2}}^{t} \frac{f_{r}(s)}{s} d s\right) d t ; x \in(0, b)
$$

then

$$
\left.\sum_{r=1}^{n} \int_{0}^{b} x^{-m} \frac{F_{r}^{p_{r}}(x)}{w_{r}^{q_{r}}(x)} \frac{F_{r}^{p_{r}+1}(x)}{w_{r}^{q_{r}+1}(x)} d x \leqslant \sum_{r=1}^{n} \frac{2 p_{r} \alpha_{r}}{m-1}\right)^{4 p_{r}} \int_{0}^{b} x^{-m w^{-2 q_{r}}(x)}\left[f_{r}(x)-f_{r}\left(\frac{x}{4}\right)\right]^{2 p_{r}} d x
$$

Proof. Using the inequality [7]

$$
\sum_{r=1}^{n} C_{r}^{p_{r}} C_{r+1}^{p_{r+1}} \leqslant \sum_{r=1}^{n} C_{r}^{2 p_{r}}
$$

where $C_{n+1}^{p_{r+1}}=C_{1}^{p_{1}}$ (for $C_{r}, 1 \leqslant r \leqslant n$ are real) and $p_{r}>1$, we observe that

$$
\sum_{r=1}^{n} \frac{F_{r}^{p_{r}}(x)}{w_{r}^{q_{r}}(x)} \frac{F_{r}^{p_{r}+1}(x)}{w_{r}^{q_{r}+1}(x)} \leqslant \sum_{r=1}^{n} \frac{F_{r}^{2 p_{r}}(x)}{w_{r}^{2 q_{r}}(x)}
$$

where $\frac{F_{r}^{p_{r}+1}(x)}{w_{r}^{q_{r}+1}(x)}=\frac{F_{1}^{p_{1}}(x)}{w_{1}^{q_{1}}(x)}, C_{r}^{p_{r}}=\frac{F_{r}^{p_{r}}(x)}{w_{r}^{q_{r}}(x)}$, and $C_{r+1}^{p_{r+1}}=\frac{F_{r}^{p_{r}+1}(x)}{w_{r}^{q_{r}+1}(x)}$.

Multiplying both sides of (2.3) by $x^{-m}$ and integrating from 0 to $b$, we obtain

$$
\sum_{r=1}^{n} \int_{0}^{b} x^{-m} \frac{F_{r}^{p_{r}}(x)}{w_{r}^{q_{r}}(x)} \frac{F_{r}^{p_{r}+1}(x)}{w_{r}^{q_{r}+1}(x)} d x \leqslant \sum_{r=1}^{n} \int_{0}^{b} x^{-m} \frac{F_{r}^{2 p_{r}}(x)}{w_{r}^{2 q_{r}}(x)} d x
$$

Integrating $\int_{0}^{b} x^{-m} \frac{F_{r}^{2 p r}(x)}{w_{r}^{2 q r}(x)} d x$ by parts, we observe that

$$
\left[1+\frac{2 q_{r} x}{m-1} \frac{w_{r}^{\prime}(x)}{w_{r}(x)} d x\right] \int_{0}^{b} x^{-m} \frac{F_{r}^{2 p_{r}}(x)}{w_{r}^{2 q_{r}}(x)} d x \leqslant \frac{2 p_{r}}{m-1} \int_{0}^{b} x^{-m} \frac{F_{r}^{2 p_{r}-1}(x)}{w_{r}^{2 q_{r}}(x)}\left(\int_{\frac{x}{4}}^{x} \frac{f_{r}(s)}{s} d s\right) d x .
$$

Since $m>1$, from (2.1) and applying Hölder's inequality [2] on (2.5) we have

$$
\int_{0}^{b} x^{-m} \frac{F_{r}^{2 p_{r}}(x)}{w_{r}^{2 q_{r}}(x)} d x \leqslant\left(\frac{2 p_{r} \alpha_{r}}{m-1}\right)^{2 p_{r}} \int_{0}^{b} x^{-m} \frac{1}{w_{r}^{2 q_{r}}(x)} R_{r}^{2 p_{r}}(x) d x,
$$

where

$$
R_{r}(x)=\int_{\frac{x}{4}}^{x} \frac{f_{r}(s)}{s} d s
$$

with $1 \leqslant \mathrm{r} \leqslant \mathrm{n}$. 
Again integrating $\int_{0}^{b} x^{-m} \frac{R_{r}^{2 p_{r}}(x)}{w_{r}^{2 q_{r}}(x)} d x$ by parts, we observe that

$$
\begin{aligned}
\int_{0}^{b} x^{-m} \frac{R_{r}^{2 p_{r}}(x)}{w_{r}^{2 q_{r}}(x)} d x= & \frac{b^{-m+1}}{-m+1} \frac{R_{r}^{2 p_{r}}(b)}{w_{r}^{2 q_{r}}(b)} \\
& -\frac{1}{-m+1} \int_{0}^{b} x^{-m+1}\left[\left(2 p_{r}\right) R_{r}^{2 p_{r}-1}(x) w_{r}^{-2 q_{r}}(x) \frac{1}{x}\left[f_{r}(x)-f_{r}\left(\frac{x}{4}\right)\right]\right. \\
& \left.-\left(2 q_{r}\right) \operatorname{Rr}^{2 p_{r}}(x) w_{r}^{-2 q_{r}-1}(x) w_{r}^{\prime}(x)\right] d x .
\end{aligned}
$$

Following the same steps from (2.5)-(2.7) with suitable modifications on (2.8), we get

$$
\int_{0}^{b} x^{-m} \frac{R_{r}^{2 p_{r}}(x)}{w_{r}^{2 q_{r}}(x)} d x \leqslant\left(\frac{2 p_{r} \alpha_{r}}{m-1}\right)^{2 p_{r}} \int_{0}^{b} x^{-m} \frac{1}{w_{r}^{2 q_{r}}(x)}\left[f_{r}(x)-f_{r}\left(\frac{x}{4}\right)\right]^{2 p_{r}} d x .
$$

From (2.4), (2.6), and (2.8), we get the required inequality (2.2).

Theorem 2.2. Let $\mathrm{p}_{\mathrm{r}}>1: 1 \leqslant \mathrm{r} \leqslant 2, \mathrm{~m}>1$ and $\mathrm{q}>0$ be a constant, $\mathrm{f}_{\mathrm{r}}$ be a nonnegative and integrable function on $(0, \mathrm{~b}), 0<\mathrm{b}<\infty$, and $w_{\mathrm{r}}$ be a positive and absolutely continuous function on $(0, \mathrm{~b})$ and

$$
1+\frac{\mathrm{q}_{\mathrm{r}} M_{1} \times}{\mathrm{m}-1} \frac{w^{\prime}(x)}{w(x)} \geqslant \frac{1}{\beta_{\mathrm{r}}} ; 1 \leqslant \mathrm{r} \leqslant 2
$$

for almost all $\mathrm{r} \in(0, \mathrm{~b})$ and for some $\beta_{\mathrm{r}}>0$. If $\mathrm{F}_{\mathrm{r}}$ is defined by

$$
F_{r}(x)=\int_{0}^{x} \frac{1}{t}\left(\int_{\frac{t}{2}}^{t} \frac{f_{r}(s)}{s} d s\right) d t ; x \in(0, b),
$$

then

$$
\begin{aligned}
& \int_{0}^{b} x^{-m}\left[\frac{F_{1}^{p_{1}}(x)}{w_{1}^{q_{1}}(x)} \frac{F_{2}^{p_{2}}(x)}{w_{2}^{q_{2}}(x)}\right]^{m_{2}}\left[\frac{F_{1}^{p_{1}}(x)}{w_{1}^{q_{1}}(x)} \frac{F_{2}^{p_{2}}(x)}{w_{2}^{q_{2}}(x)}\right]^{m_{1}} d x \\
& \leqslant 2^{m_{1}-1} \sum_{r=1}^{2}\left(\frac{p_{r} M_{1} \beta_{r}}{m-1}\right)^{2 p_{r} M_{1}} \int_{0}^{b} x^{-m} w^{-q_{r} M_{1}}(x)\left[f_{r}(x)-f_{r}\left(\frac{x}{2}\right)\right]^{p_{r} M_{1}} d x
\end{aligned}
$$

where $M_{1}=m_{1}+2 m_{2}$.

Proof. Using the inequality [6]

$$
\left[C_{1} C_{2}\right]^{m_{2}}\left[C_{1} C_{2}\right]^{m_{1}} \leqslant 2^{m_{1}-1}\left[C_{1}^{M_{1}}+C_{2}^{M_{1}}\right]
$$

where $C_{1}$ and $C_{2}$ are positive reals, $m_{1}$ and $m_{2}$ are positive integers, and $M_{1}=m_{1}+2 m_{2}$, we observe that

$$
\left[\frac{F_{1}^{p_{1}}(x)}{w_{1}^{q_{1}}(x)} \frac{F_{2}^{p_{2}}(x)}{w_{2}^{q_{2}}(x)}\right]^{m_{2}}\left[\frac{F_{1}^{p_{1}}(x)}{w_{1}^{q_{1}}(x)} \frac{F_{2}^{p_{2}}(x)}{w_{2}^{q_{2}}(x)}\right]^{m_{1}} \leqslant 2^{m_{1}-1}\left[\frac{F_{1}^{p_{1} M_{1}}(x)}{w_{1}^{q_{1} M_{1}}(x)}+\frac{F_{2}^{p_{2} M_{1}}(x)}{w_{2}^{q_{2} M_{1}}(x)}\right],
$$

where $C_{1}=\frac{F_{1}^{P_{1}}(x)}{w_{1}^{q_{1}}(x)}$ and $C_{2}=\frac{F_{2}^{P_{2}}(x)}{w_{2}^{q_{2}}(x)}$.

Multiplying both sides of (2.11) by $x^{-m}$ and integrating from 0 to $b$, we obtain

$$
\begin{aligned}
& \int_{0}^{b} x^{-m}\left[\frac{F_{1}^{p_{1}}(x)}{w_{1}^{q_{1}}(x)} \frac{F_{2}^{p_{2}}(x)}{w_{2}^{q_{2}}(x)}\right]^{m_{2}}\left[\frac{F_{1}^{p_{1}}(x)}{w_{1}^{q_{1}}(x)} \frac{F_{2}^{p_{2}}(x)}{w_{2}^{q_{2}}(x)}\right]^{m_{1}} d x \\
& \leqslant 2^{m_{1}-1}\left[\int_{0}^{b} x^{-m} \frac{F_{1}^{p_{1} M_{1}}(x)}{w_{1}^{q_{1} M_{1}}(x)} d x+\int_{0}^{b} x^{-m} \frac{F_{2}^{p_{2} M_{1}}(x)}{w_{2}^{q_{2} M_{1}}(x)} d x\right],
\end{aligned}
$$


where $M_{1}=m_{1}+2 m_{2}$. Let us consider both the integrals on the right side of (2.12) simultaneously. Integrating $\int_{0}^{b} x^{-m} \frac{F_{1}^{p_{1} M_{1}}(x)}{w_{1}^{q_{1} M_{1}}(x)} d x$ by parts, we observe that

$$
\left[1+\frac{q_{1} M_{1} x}{m-1} \frac{w_{1}^{\prime}(x)}{w_{1}(x)} d x\right] \int_{0}^{b} x^{-m} \frac{F_{1}^{p_{1} M_{1}}(x)}{w_{1}^{q_{1} M_{1}}(x)} d x \leqslant \frac{p_{1} M_{1}}{m-1} \int_{0}^{b} x^{-m} \frac{F_{1}^{p_{1} M_{1}-1}(x)}{w_{1}^{q_{1} M_{1}}(x)}\left(\int_{\frac{x}{2}}^{x} \frac{f_{1}(s)}{s} d s\right) d x .
$$

Since $m>1$, from (2.9) and applying Hölder's inequality [2] on (2.13), we have

$$
\int_{0}^{b} x^{-m} \frac{F_{1}^{p_{1} M_{1}}(x)}{w_{1}^{q_{1} M_{1}}(x)} d x \leqslant\left(\frac{p_{1} M_{1} \beta_{1}}{m-1}\right)^{p_{1} M_{1}} \int_{0}^{b} x^{-m} \frac{1}{w_{r}^{q_{1} M_{1}}(x)} R_{1}^{p_{1} M_{1}}(x) d x,
$$

where

$$
R_{1}(x)=\int_{\frac{x}{2}}^{x} \frac{f_{1}(s)}{s} d s
$$

Again integrating $\int_{0}^{b} x^{-m} \frac{R_{1}^{p_{1} M_{1}}(x)}{w_{1}^{q_{1} M_{1}}(x)} d x$ by parts, we observe that

$$
\begin{aligned}
\int_{0}^{b} x^{-m} \frac{R_{1}^{p_{1} M_{1}}(x)}{w_{1}^{q_{1} M_{1}}(x)} d x= & \frac{b^{-m+1}}{-m+1} \frac{R_{1}^{p_{1} M_{1}}(b)}{w_{1}^{q_{1} M_{1}}(b)} \\
& -\frac{1}{-m+1} \int_{0}^{b} x^{-m+1}\left[\left(p_{1} M_{1}\right) R_{1}^{p_{1} M_{1}-1}(x) w_{1}^{-q_{1} M_{1}}(x) \frac{1}{x}\left[f_{1}(x)-f_{1}\left(\frac{x}{2}\right)\right]\right. \\
& \left.-\left(q_{1} M_{1}\right) R_{1}^{p_{1} M_{1}}(x) w_{1}^{-q_{1} M_{1}-1}(x) w_{1}^{\prime}(x)\right] d x .
\end{aligned}
$$

Following the same steps from (2.13)-(2.14) with suitable modifications on (2.16), we get

$$
\int_{0}^{b} x^{-m} \frac{R_{1}^{p_{1} M_{1}}(x)}{w_{1}^{q_{1} M_{1}}(x)} d x \leqslant\left(\frac{p_{1} M_{1} \beta_{1}}{m-1}\right)^{p_{1} M_{1}} \int_{0}^{b} x^{-m} \frac{1}{w_{1}^{q_{1} M_{1}}(x)}\left[f_{1}(x)-f_{1}\left(\frac{x}{2}\right)\right]^{p_{1} M_{1}} d x .
$$

From (2.14) and (2.17), we have

$$
\int_{0}^{b} x^{-m} \frac{F_{1}^{p_{1} M_{1}}(x)}{w_{1}^{q_{1} M_{1}}(x)} d x \leqslant\left(\frac{p_{1} M_{1} \beta_{1}}{m-1}\right)^{2 p_{1} M_{1}} \int_{0}^{b} x^{-m_{w}} w^{-q_{1} M_{1}}(x)\left[f_{1}(x)-f_{1}\left(\frac{x}{2}\right)\right]^{p_{1} M_{1}} d x .
$$

Similarly integrating $\int_{0}^{b} x^{-m} \frac{F_{2}^{p_{2} M_{1}}(x)}{w_{2}^{q_{2} M_{1}}(x)} d x$ by parts, we get

$$
\begin{aligned}
\int_{0}^{b} x^{-m} \frac{F_{2}^{p_{2} M_{1}}(x)}{w_{2}^{q_{2} M_{1}}(x)} d x= & \frac{b^{-m+1}}{-m+1} \frac{F_{2}^{p_{2} M_{1}}(b)}{w_{2}^{q_{2} M_{1}}(b)}-\frac{1}{-m+1} \int_{0}^{b} x^{-m+1}\left[\left(p_{2} M_{1}\right) F_{2}^{p_{2 M_{1}}-1}(x) w_{2}^{-q_{2} M_{1}}(x) F_{2}^{\prime}(x)\right. \\
& \left.-\left(q_{2} M_{1}\right) F_{2}^{p_{2} M_{1}}(x) w_{2}^{-q_{2} M_{1}-1}(x) w_{2}^{\prime}(x)\right] d x .
\end{aligned}
$$

The rest of the proof follows from (2.13)-(2.15) with suitable changes, therefore

$$
\int_{0}^{b} x^{-m} \frac{F_{2}^{p_{2} M_{1}}(x)}{w_{2}^{q_{2} M_{1}}(x)} d x \leqslant\left(\frac{p_{2} M_{1} \beta_{1}}{m-1}\right)^{2 p_{2} M_{1}} \int_{0}^{b} x^{-m_{1}} w^{-q_{2} M_{1}}(x)\left[f_{2}(x)-f_{2}\left(\frac{x}{2}\right)\right]^{p_{2} M_{1}} d x .
$$

From (2.12), (2.18), and (2.19) we get the desired inequality (2.10).

\section{References}

[1] G. H. Hardy, Note on a theorem of Hilbert, Math. Z., 6 (1920), 314-317. 1, 1

[2] G. H. Hardy, J. E. Littlewood, G. Pólya, Inequalities, 2d ed., Cambridge, at the University Press, (1952). 2, 2

[3] M. Izumi, S.-I. Izumi, On some inequalities for Fourier series, J. Analyse Math., 21 (1968), 277-291. 1, 1 
[4] N. Levinson, Generalizations of an inequality of Hardy, Duke Math. J., 31 (1964), 389-394. 1

[5] D. S. Mitronović, Analytic Inequalities, In cooperation with P. M. Vasić, Die Grundlehren der mathematischen Wissenschaften, Band 165 Springer-Verlag, New York-Berlin, (1970).

[6] B. G. Pachpatte, On a new class of Hardy type inequalities, Proc. Roy. Soc. Edinburgh Sect. A, 105 (1987), 265-274. 2

[7] S. Salem, On some integral inequalities of Hardy type, Kyungpook Math. J., 35 (1995), 193-203. 2

[8] M. Z. Sarikaya, H. Yildirim, Some Hardy type integral inequalities, JIPAM. J. Inequal. Pure Appl. Math., 7 (2006), 5 pages.

[9] B. Sroysang, More on some Hardy type integral inequalities, J. Math. Inequal., 8 (2014), 497-501. 1 